\title{
Essais
}

Revue interdisciplinaire d'Humanités

Hors-série 7 | 2022

Récits d'outre-thèse

\section{Une éducation doctorale}

\author{
A doctoral education
}

Nina Mansion

\section{(2) OpenEdition \\ 1 Journals}

Édition électronique

URL : https://journals.openedition.org/essais/10875

DOI : 10.4000/essais. 10875

ISSN : 2276-0970

Éditeur

École doctorale Montaigne Humanités

\section{Édition imprimée}

Date de publication : 15 avril 2022

ISBN : 979-10-970024-00-0

ISSN : 2417-4211

\section{Référence électronique}

Nina Mansion, « Une éducation doctorale », Essais [En ligne], Hors-série 7 | 2022, mis en ligne le 01 février 2022, consulté le 19 janvier 2023. URL : http://journals.openedition.org/essais/10875 ; DOI https://doi.org/10.4000/essais.10875

Ce document a été généré automatiquement le 19 janvier 2023.

Tous droits réservés 


\title{
Une éducation doctorale
}

\author{
A doctoral education
}

Nina Mansion

1 La lecture de l'appel à contributions pour une publication portant sur le devenir des docteurs suscita tout de suite mon intérêt. L'opportunité m'était donnée de revenir sur mon parcours, quelques mois seulement après ma soutenance et mon recrutement. De mettre distance et critique, là où la fulgurance des dernières semaines avait emporté avec elle toute possibilité d'examiner le sens de cette trajectoire inattendue. Mais les mots ont tardé à venir. Trop de choses à dire, peut-être. Un sujet trop personnel pour que les tournures scolaires et certainement jargonnantes que j'avais l'habitude de mobiliser suffisent. Comme devant un édifice installé sur une place trop étroite, je ne trouvais pas le recul nécessaire. Surement aussi parce que l'écriture est un sport qu'il faut pratiquer avec une grande régularité. Or, je suis au repos depuis un petit moment. Depuis que je suis devenue docteure. Avant cette pause, j'avais l'impression d'avoir acquis une forme d'aisance. Mon esprit était comme échauffé. L'effort n'était presque plus perceptible. Les phrases glissaient de mes doigts jusqu'à l'écran. Mes pensées prenaient forme naturellement, l'objectif m'apparaissait clairement. Aujourd'hui, les mots butent. Les lettres s'affichent puis s'effacent presque aussi vite.

2 Je suis docteure depuis un an et demi. Ou plus exactement depuis quinze mois. Quinze mois pendant lesquels la terre a fait une fois et demi le tour du soleil et pendant lesquels ma vie a connu elle aussi une révolution. Des révolutions. En un an, il s'est passé plus de choses que je n'aurais accepté de le croire si quiconque s'était risqué à me prédire mon avenir. Une soutenance, une naissance, une pandémie, un recrutement, et un certain chaos. Cependant, outre le récit du chemin parcouru depuis la soutenance, je formule le vœu que ce texte sonne aussi comme un encouragement aux doctorants au travail et aux docteurs en quête d'un poste. En effet, parmi les voix qui vont s'exprimer sur l'avenir des docteurs en sciences humaines et sociales, la mienne sera certainement dissonante. Je n'allongerai pas les vers de la complainte - ô combien légitime - du jeune docteur. Car ma trajectoire est celle des (trop rares) chanceux. Elle est de celles qui, je 
l'espère, nourriront l'espoir des doctorants actuellement au travail. C'est, en tout cas, un des objectifs que je me fixe ici.

\section{Écrire l'histoire}

3 Je vais donc m'efforcer d'écrire mon histoire, après avoir écrit celle des autres. En effet, ma thèse, qui portait sur l'invention d'une politique de collecte des archives des architectes en France au milieu des années $1970^{1}$, raconte l'histoire d'une poignée d'acteurs - architectes, historiens de l'art et figures politiques - qui ont œuvré à la protection de ces documents longtemps ignorés. À travers une série d'entretiens, j'ai cherché à cerner des démarches, à dessiner des portraits, à comprendre des parcours. Outre le prétexte des archives des architectes, c'est l'histoire de l'architecture qui m'importait. Son histoire, ses acteurs. Dans une mise en abyme et en histoire du métier auquel je me destinais, j'ai voulu comprendre ce qu'était, hier et aujourd'hui, un historien de l'architecture. Ayant pour objet ma propre discipline, mon travail de thèse s'intéressait donc à ceux qui l'avaient façonnée au cours des dernières décennies. C'est dans cette logique que l'historien de l'architecture et le conservateur des archives qui dirigaient ma thèse en sont devenus aussi des protagonistes. Aucun hasard là-dedans. C'est après avoir entendu le récit de leur expérience commune à la tête d'une institution chargée de collecter les archives des architectes que j'ai décidé de consacrer mes recherches à ces dernières. La difficulté alors n'était pas tant la mise à distance chronologique d'évènements relativement récents que la mise à distance critique de discours prononcés par des personnes proches de moi. Les tenants de l'histoire immédiate et ceux de l'histoire du temps présent ont ouvert la voie. Ils ont montré qu'il n'était pas toujours plus aisé de prendre du recul sur un évènement s'étant déroulé il y a un siècle que sur celui datant d'hier. D'autres, comme Yvan Jablonka qui a mené une enquête sur la trajectoire de ses grands-parents juifs polonais immigrés à Paris avant d'être déportés à Auschwitz ${ }^{2}$, ont rendu possible l'écriture d'une histoire à la fois personnelle et scientifique. Armée de ces lectures et références, je me suis autorisée une certaine proximité, voire une promiscuité avec mon sujet de recherche. L'histoire de ma thèse et mon histoire tant personnelle que professionnelle sont donc intrinsèquement mêlées depuis le départ. Ce texte ne dérogera pas à ce principe.

\section{Le privilège des privilégiés (le doctorat)}

4 J'ai soutenu ma thèse de doctorat le 28 novembre 2019. Ce fut une très belle journée, une des meilleures. Je faisais aboutir un travail long de presque sept années et je portais dans mon ventre, déjà bien arrondi, mon premier enfant. Entourée de mes proches, je savourais sereinement cet aboutissement. J'avais - déjà - le sentiment d'être privilégiée. J'avais mené ma thèse dans des conditions idéales : après une petite année de galère, je décrochais au début de ma deuxième année un contrat doctoral. Outre la garantie d'une stabilité financière pendant quelques années, ce contrat devait m'apporter le cadre professionnel et l'environnement social qui me manquait. Après un premier échec, je consacrais donc ma première année de thèse à la préparation d'un projet abouti et réaliste. Entre mes deux tentatives, les règles du jeu furent repensées. Plutôt qu'une sélection effectuée au sein de la douzaine de laboratoires rattachés à l'école doctorale, elle serait désormais menée, de manière collégiale et transparente, 
par un jury constitué des directeurs de laboratoires, d'invités extérieurs, de la direction de l'école. En atteignant ce graal, j'entrais, en effet, dans le cercle très privilégié des "financés », ceux qui se paient le luxe de ne pas avoir à mener de front la rédaction d'une thèse et les contraintes d'un boulot alimentaire. Le sentiment d'être privilégiée était évidemment corrélé à l'obtention de ce contrat qui me permettait en outre de dispenser des enseignements (charge qui, cela pourra surprendre, s'avère difficile d'accès), mais pas uniquement. Dès le départ, j'ai eu la chance d'être dirigée ou, plus exactement, accompagnée et soutenue dans cette vaste entreprise qu'est le doctorat par une personnalité brillante et, chose peut-être plus rare, bienveillante. Mon directeur de thèse a écrit et construit cette trajectoire avec moi. Ma thèse fut la première qu'il menait à soutenance. Je ne me livre pas ici à l'expression convenue de remerciements attendus à l'endroit de mon directeur de thèse. Je lui suis sincèrement reconnaissante et je pense que mon parcours lui doit beaucoup. À son contact, je suis devenue historienne. J'y ai notamment appris le « respect de la juste distance que [l'historien] se doit d'entretenir avec son objet d'étude ${ }^{3}$ » et le sens d'une éthique professionnelle. L'instauration d'une relation de confiance, faite d'échanges réguliers, d'exigence et de soutien, entre un directeur de recherche et un doctorant n'est pas une évidence.

5 Sa présence ne fut pas la seule importante. En effet, il me semble que j'ai eu une autre chance : celle d'être bien entourée. Mon expérience donne tort au récit du thésard solitaire et isolé. Loin de moi la volonté de nier cette réalité. Mais, il se trouve qu'à partir de ma deuxième année de thèse, le doctorat est devenu pour moi une expérience collective. Cette situation doit beaucoup à l'école doctorale dans laquelle je me suis inscrite en entamant cette thèse. Bien sûr, je me suis efforcée tôt de donner vie à un environnement social bienveillant, de tisser un réseau amical au sein de mes collègues doctorants, de m'investir dans toute forme de projets collectifs. Néanmoins, ce n'est que lors d'évènements organisés par cette école doctorale, à l'instar du raout annuel que constitue la « rentrée de l'école doctorale » que j'ai fait la connaissance de ceux qui deviendraient mes amis, mes alliés. Selon leur acception administrative, les écoles doctorales délivrent le doctorat, en prenant soin de vérifier qu'il est réalisé dans le cadre défini par la loi, et dispensent une formation. Celle que j'ai fréquentée était aussi un lieu, un «lieu social» pour reprendre une notion formulée par Michel de Certeau qui a nourri mes analyses. C'est là que je suis devenue chercheuse. Sans véritablement m'en rendre compte, un peu plus chaque jour que je passais dans la «salle des doctorants ». Dans le cadre de mes recherches de thèse, je me suis attachée à analyser les effets du lieu physique de production de la recherche sur la construction d'une discipline et les phénomènes de professionnalisation des sciences humaines. En m'inspirant des premières recherches de Bruno Latour sur "la vie de laboratoire ${ }^{4}$ " notamment, j'ai cherché à comprendre si la mise en œuvre d'un centre de conservation des archives des architectes dans lequel de jeunes architectes ou historiens pouvaient venir étudier ces même documents avait pu contribué à la reconnaissance de l'histoire de l'architecture en tant que discipline. J'ai tenté de mettre en évidence le rôle du lieu sur la professionnalisation de l'histoire de l'architecture en montrant le pouvoir de mise en réseau de ce centre d'archives dans lequel se définit une part des règles et des normes charpentant la discipline. Il me semble que la «Maison de la Recherche» de mon université a eu un effet analogue sur mes camarades et moi. Je ne prétends pas qu'il en fut de même pour tous les doctorants de l'école doctorale à laquelle j'appartenais. Néanmoins, pour quelques-uns d'entre nous elle fut un de ces lieux du 
partage de connaissances et de compétences, de discussions et de disputes; un de ces lieux où se construit la recherche et où grandissent les chercheurs.

Dans ce contexte favorable, je me suis doucement attelée, parallèlement à mes recherches, à façonner le "CV parfait». Car le doctorat n'est pas la thèse, et inversement. Le doctorat est beaucoup plus que cela. Ce fut très vite mon sentiment, en tout cas. Je confesse sans mal avoir développé tôt un certain sens de la stratégie. L'écosystème de jeunes chercheurs qui m'entourait à la "salle des doctorants " y est pour beaucoup. À chaque pause café, à chaque déjeuner, nous évoquions nos candidatures refusées à un colloque portant précisément sur notre domaine d'expertise, les commentaires alambiqués reçus pour un article soumis trois ans plus tôt, les atermoiements autour de la constitution de notre jury de soutenance mais aussi les règles rédactionnelles des notes de bas de page, la mise en page des annexes ou nos rétroplanning de rédaction. Tout y est passé. Années après années, j'ai donc participé, comme il se devait, à de nombreux évènements scientifiques. D'abord, des journées d'étude. Puis, des colloques nationaux et, un peu plus tard, internationaux. Ensuite, j'en ai organisés. Parallèlement, je publiais mes premiers articles. Bonne élève, j'ai fait les choses dans l'ordre. Cherchant des opportunités de travailler de manière collective, je me suis investie dans le comité de rédaction de la revue de l'école doctorale. À travers ces diverses expériences et au contact de mes camarades, je me familiarisais avec le cadre légal et professionnel dans lequel évoluent les doctorants. C'est donc naturellement que je me suis portée candidate en tant que représentante des doctorants au sein de mon laboratoire d'abord, puis de la Commission Recherche de l'Université. Parallèlement à ces expériences locales, j'ai voulu m'investir auprès de l'Association d'Histoire de l'Architecture (AHA) dont je suis vice-présidente Doctorants. Il ne s'agissait pas d'enchaîner les missions, mais de saisir les opportunités offertes par le doctorat pour me former au mieux au métier d'enseignant-chercheur.

7 Au terme des trois années de contrat doctoral, j'ai eu la chance de pouvoir défendre ma candidature pour un poste d'ATER, que j'ai obtenu. Cette logique, qui consiste à donner à ceux qui ont déjà eu, à soutenir les privilégiés si l'on veut, m'a évidemment bénéficié. Mais, à partir de cette période, les engagements pris dans le cadre de ces contrats, à savoir dispenser des enseignements et contribuer à la recherche, prenaient nettement le pas sur mes recherches. Ma thèse accusait un retard certain. Mener une vie d'enseignant-chercheur en formation - car c'est comme cela que j'entends le doctorat s'avère difficilement compatible avec l'intense labeur que représente la rédaction d'une thèse. D'autant plus lorsqu'il s'agit d'une thèse en sciences humaines et sociales, comptant rarement moins de 400 pages (dans mon cas, il s'agit de 690 pages d'analyse et presque autant d'annexes) et totalisant généralement une petite demi-douzaine d'années de travail (mon bilan s'établit à 75 mois, soit un peu plus de six ans). Cette activité, proprement solitaire, exigeante et absorbante, s'accommode mal de distractions extérieures, même les plus scientifiques d'entre elles. Et que dire d'une vie de famille?

8 À la rentrée 2018, ayant épuisé les différentes modalités de financements doctoraux et bien décidée à terminer cette thèse, je faisais donc le choix de "pointer à Pôle Emploi ", comme on dit. Pendant une année et demi, ma vie s'est limitée à écrire et à dormir. Résumée pour les besoins de l'exercice, cette assertion n'est pas si éloignée de la réalité. Je me suis, d'ailleurs, récemment remémoré ces souvenirs à la saveur si particulière à la lecture d'un petit roman qui parlera à beaucoup d'entre nous, La soutenance d'Anne 
Urbain. À la différence de son héros (Antoine, jeune thésard occupé à interroger la censure dans la littérature érotique) et à ma plus grande surprise, la "rédaction » est un bon souvenir. C'est peut-être un des premiers enseignements de cet Essai rétrospectif : ce qui nous avait été présenté, depuis notre inscription en thèse, comme la plus grande des épreuves s'est révélé en constituer le climax, l'apogée. Les mois d'interrogation s'ancraient dans le passé, les doutes s'éloignaient, les obstacles se dispersaient. L'écriture apportait la lumière. Les mots donnaient forme à l'informe. De nature profondément anxieuse, je vivais la rédaction sans l'apparition de symptômes inexpliqués, de maladies imaginaires et autres maux caractéristiques des personnalités de mon espèce. Ce fut donc une période épanouissante qui trouva une conclusion logique dans une soutenance que je me risquerais à qualifier de réussie: un jury prestigieux, des échanges fertiles, mes proches autour de moi réunis. J'étais donc docteure. Heureuse. Délestée d'un poids. Pas du tout préparée à ce qui allait suivre.

\section{Le mirage de la « success story » (l'après-thèse)}

9 Quelques mois plus tard, une pandémie éclatait alors que je venais de donner naissance à ma fille. Le bouleversement cataclysmique engendré par l'arrivée d'un premier enfant et le spectre d'un virus poison inconnu qui allait mettre le monde entier à l'arrêt et sans dessus-dessous ont rapidement fait de l'euphorie de la soutenance et des satisfactions d'un doctorat finalement achevé de lointains souvenirs. Je pénétrais alors dans un long tunnel dont je ne ressortirai que quelques mois plus tard. Alors que mon entourage se réjouissait pour moi, je ne parvenais pas à remonter à la surface, aspirée par le courant puissant d'un mal dont j'étais la première surprise. Si la thèse ou son terme n'en sont pas la cause, il serait naïf de penser que la transition d'un état de folle émulation intellectuelle rythmé de gratifications régulières et d'intenses stimulations à celui de profonde inertie qu'imposait un confinement d'ampleur nationale couplé à une inactivité professionnelle (expliquée à la fois par mon congé maternité et le dépôt de la thèse) n'aurait rien à voir avec tout cela. Alors que j'avais longuement attendu ce moment, je ne parvenais pas faire le deuil de la gestation intellectuelle et individuelle qu'a constitué pour moi la thèse. Si je m'autorise à évoquer ici cet épisode intime de ma vie de docteure, c'est parce qu'il me semble que la problématique de la santé psychique des jeunes chercheurs, qu'ils soient doctorants ou docteurs, est trop rarement discutée. Combien sommes-nous à avoir trainé nos guêtres dans les salles d'attente de psychiatres, psychanalystes ou thérapeutes d'un autre type ? Combien ont ajouté leurs noms aux longues listes d'attente des Centre médicaux-psychologiques des grandes métropoles universitaires? Les errements et égarements psychologiques des thésards ont été évoqués par d'autres avant moi $^{5}$ et le dossier de la revue Essais auquel ce texte entend contribuer apportera, je l'espère, une nouvelle pierre à cet édifice qu'il me semble aujourd'hui nécessaire consolider.

10 Les première sources de lumière prirent une forme inattendue : les candidatures aux postes de maître de conférences. Avec elles vinrent la possibilité d'une projection, la possibilité d'une vie après. Ces impératifs professionnels furent une respiration. Une profonde inspiration à un moment où j'étouffais sous le poids des responsabilités, de la fatigue et des doutes qu'avait amené avec lui mon statut nouvellement acquis de mère. Aussi comblée étais-je, je ne me reconnaissais plus tout à fait. De doctorante enthousiaste, dynamique et ambitieuse, j'étais devenue docteure sans emploi et dans 
l'impossibilité circonstancielle d'en projeter un, alors que les universités avaient fermé leurs portes pour une période indéterminée. Alors, quand les premières annonces de poste ont été publiées, l'angoisse de l'enfermement (physique et psychique) et l'incertitude vis-à-vis de nos avenirs s'allégèrent. Ces candidatures me donnaient l'illusion d'une vie qui allait reprendre son cours presque normalement. En réalité, rien ne disait si les procédures de recrutement allaient pouvoir se tenir comme prévues. Mais il était rassurant de prétendre que les enjeux restaient inchangés. Je me plongeais alors entièrement dans ce processus prospectif.

11 Dans un enchaînement d'évènements que je ne m'explique toujours pas, je fus finalement recrutée en tant que maître de conférence des écoles nationales supérieures d'architecture à l'été 2020. Les universités furent les premières à ouvrir le grand cérémonial des recrutements. Après l'euphorie, la déconvenue. Aucune offre ne correspondait à mon profil. Une seule annonce retint mon attention : en tirant bien de tous les côtés, j'allais peut-être pouvoir faire croire que j'avais certaines des compétences requises. Quelques semaines plus tard, les écoles d'architecture, qui ont harmonisé leurs statuts à ceux de l'université très récemment, ont fait connaître leurs besoins. Dans le champ Histoire et cultures architecturales (les champs sont des équivalents des sections du $\mathrm{CNU}$ ), neuf postent furent ouverts cette année-là. À partir de cette phase là, j'ai « activé mon réseau ", comme on dit. J'ai cherché à comprendre quels postes étaient déjà fléchés, quels postes étaient ouverts à des profils différents (pour le dire plus clairement, ouvert à des non-architectes). Je décidais finalement de candidater à seulement deux d'entre eux. Les raisons en sont multiples : cohérence de mon profil par rapport à la fiche de poste, situation géographique (conciliation délicate entre attrait d'une ville où l'on s'imagine déplacer toute la famille et déplacements gérables le temps d'envisager une délocalisation) et pression de la concurrence (renoncement aux postes franciliens considérés comme inaccessibles face aux demandes importantes de «parisiens»). Mon appétence s'est vite portée sur le poste que j'occupe actuellement, qui avait l'avantage de présenter un bon équilibre entre vie professionnelle (équipe dynamique et orientations scientifiques et pédagogiques stimulantes) et vie personnelle (rapprochement familial, notamment). Je passerai les détails de la préparation de ma candidature et de mon audition à laquelle je me consacrais entièrement au printemps. J'insisterai simplement sur le fait que j'ai eu la chance d'être relue et auditionnée en amont par une cohorte de maîtres de conférence et de professeurs rencontrés pendant mon parcours et sollicités face à l'enjeu. Il me semble que ces contributions furent décisives. Au regard du contexte sanitaire défavorable, les auditions furent organisées sur Zoom. Même si un puissant orage ayant coupé la ligne internet a bien failli me couter ma deuxième audition, j'ai finalement trouvé du bon à cette situation qui permet le confort de son environnement personnel dans un moment nécessitant concentration et sérénité. Enfin, il va sans dire que la mobilité fut un enjeu déterminant de cette procédure de recrutement. La nécessité de changer de lieu de vie lorsqu'un poste intéressant se présenterait étant un postulat acquis dans notre couple depuis de longues années, le déménagement de notre famille dans une nouvelle région s'est donc fait naturellement, mais non moins facilement. Quinze mois après ma soutenance, nous posions nos cartons dans une nouvelle maison, une ville inconnue jusque là alors que je faisais progressivement la connaissance de mes collègues et des étudiants, tout cela par écrans interposés. 


\section{Retour critique}

12 Lorsque je me suis engagée dans le doctorat, j'étais pétrie des plus naïves ambitions, espérant devenir rapidement professeur à l'université. Le jargon académique m'était encore complètement étranger. Je n'avais jamais entendu parlé du CNU, encore moins de l'HCERES ou des projets financés par l'ANR et l'ERC. Je pensais, par exemple, qu'il suffisait de soutenir sa thèse pour devenir maître de conférence et ignorait tout du processus de la "qualification », cette spécificité française certes garante d'une forme de tradition disciplinaire, mais surement dépassée. Ce vocabulaire, je l'ai acquis à l'école doctorale au cours de formations, mais aussi et surtout au fil d'échanges informels - ceux-là même qui à l'heure de la pandémie doivent faire terriblement défaut aux doctorants - autour d'un café pendant les longues journées d'écriture ou d'une boisson plus festive lors des colloques et soutenances de thèse, qui sont des moments fondateurs de la vie d'un jeune chercheur. Ma crédulité fut rapidement douchée par de très régulières mises en garde. Les récits du docteur "en galère ", enchaînant les contrats de vacation et les jobs alimentaires, dessinaient pour mes collègues et moi un horizon peu reluisant, auquel il nous paraissait alors difficile d'échapper. Parmi mes connaissances proches ou plus lointaines, certains s'inquiétaient de me voir prendre cette voie peu lucrative, après un parcours scolaire pourtant brillant. Pour d'autres, j'appartenais automatiquement à cette caste des intellectuels qui, pourtant payés avec «les sous du contribuable », peinent à contribuer concrètement à la bonne santé sociale et économique du pays. En effet, je n'ai pas choisi le parcours le plus simple ou le plus évident. J'aurais peut-être pu devenir avocate (première ambition, abandonnée dès les portes ouvertes de la faculté de droit), diplomate ou grand-reporter. Je suis devenue historienne de l'art.

13 Être historien de l'art aujourd'hui - qui plus est de l'architecture, comme c'est mon cas - n'est pas précisément ce que l'on pourrait considérer comme un "profil porteur sur le marché de l'emploi ». Les données sur les recrutements aux postes de maître de conférences et de professeur des universités françaises ne sont pas facilement accessibles. Au cours des dernières années, les chiffres semblent se stabiliser, voire connaitre une légère augmentation pour atteindre en 2020 treize postes de MCF ouverts et 8 postes de professeurs ${ }^{6}$. Dans le champ de l'histoire de l'architecture, la situation est plus délicate à cerner, les postes étant répartis entre deux établissements d'enseignement supérieur: les départements d'histoire de l'art des universités et les écoles nationales supérieures d'architecture. Les recrutements dans les écoles d'architecture sont montés en puissance au cours des dernières années. À l'université, le bilan est édifiant: aucun recrutement sur un poste de maître de conférences en histoire de l'architecture dans les universités françaises entre 2011 et 2019. Récemment, deux maîtres de conférence ont été nommés dans un département d'histoire de l'art et dans un institut de géographie. Ces évènements traduisent-ils un rebond de l'histoire de l'architecture universitaire? La participation active des deux lauréats à l'Association d'Histoire de l'Architecture, relancée il y a quelques années, a-telle quelque chose à voir avec cette reconnaissance tardive? Il me faudrait poursuivre les analyses menées dans le cadre de ma thèse sur la professionnalisation de l'histoire de l'architecture à la fin du $\mathrm{XX}^{\mathrm{e}}$ siècle pour pouvoir apporter une réponse autre qu'une impression individuelle, une intuition personnelle. Des publications récentes, à l'instar de l'opus À quoi sert l'histoire de l'architecture ?? dirigé par Richard Klein, nous éclairent 
néanmoins sur la situation de cette discipline en voie de reconnaissance. Malgré tout, à l'échelle des postes dans l'enseignement supérieur, l'histoire de l'architecture est une niche. C'est à la fois un inconvénient (il y a très peu de postes) mais c'est aussi un avantage (une plus faible concurrence mais surtout des réseaux plus accessibles dans lesquels on peut plus facilement faire sa place).

L'histoire de l'art et celle de l'architecture sont des disciplines en voie de féminisation. Dans mon laboratoire, un des plus petits (en nombre de chercheurs) de l'université puisqu'il réunissait uniquement les chercheurs en histoire de l'art, nous avons presque chaque année un contrat doctoral, pourtant très convoité et difficile à décrocher. Je ne crois pas qu'il me revienne d'expliciter ce palmarès surprenant. Je me permets seulement de relever que, contre toute attente, tous les lauréats de ces financements furent de jeunes femmes, qu'elles ne travaillaient pas toujours sur des sujets dits "porteurs", qu'elles ne sortaient pas de grandes écoles, qu'elles n'étaient pas issues des milieux les plus favorisés. Car ce sont là des clichés qui persistent. Comme en témoigne un rapport de 2021 préparé par le ministère de l'Enseignement supérieur et de la recherche, les femmes titulaires d'un doctorat disposeraient de conditions d'emplois moins favorables que les hommes trois ans après l'obtention de leur diplôme et toucherait un salaire inférieur d'environ $8 \%^{8}$. Extraits de leur contexte, ces chiffres n'ont pas valeur. Si le nombre de femmes aux postes de maître de conférences et de professeurs est en nette augmentation dans les universités françaises (notamment dans les domaines des humanités), elles restent globalement minoritaires aux postes d'enseignant-chercheurs : elles en occupaient seulement $40 \%$ en $2019^{9}$. On observe ainsi une courbe inversée : très nombreuses parmi les étudiants, les femmes figurent en petit nombre parmi les professeurs. Le schéma s'inverse chez les hommes. La place des femmes, même dans le domaine de la recherche en sciences humaines et sociales, reste complexe et leurs trajectoires souvent jalonnées d'obstacles trop souvent ignorés. Je n'entrerais pas dans des considérations personnelles mais je me permettrais simplement d'évoquer une anecdote : ma maternité n'a posé aucun problème dans mon parcours de doctorante, mais j'ai reçu de nombreuses félicitations pour ma capacité à avoir maitrisé mon calendrier. Je n'en dirais pas plus. Le sujet de la féminisation de l'enseignement et de la recherche en sciences humaines et sociales mériterait largement un article. Il ne me revient pas de l'écrire. Quelques réflexions sur cette problématique semblaient néanmoins s'imposer.

Enfin, je m'essaierai pour terminer à un exercice de synthèse justificative, dans l'espoir de clarifier les choses pour les docteurs en devenir et les doctorants à venir. Ma trajectoire est donc celle d'une étudiante, certes sérieuse et incontestablement ambitieuse, mais qui a aussi bénéficié d'un système qui privilégie les privilégiés. Mon statut actuel est le résultat d'une détermination indéniable couplée à un magma de chances et de privilèges, ainsi qu'à une conscientisation des stratégies pertinentes, sans cesse discutées dans le contexte professionnel et dans le cercle intime. Peut-être ai-je rempli toutes les cases à cocher pour être recrutée : avoir soutenu une thèse dont le jury a reconnu les qualités, avoir su m'entourer en constituant un réseau solide, avoir publié et communiqué régulièrement sur l'avancée de mes travaux. Un élément n'a peut-être pas été suffisamment souligné jusqu'à présent: le choix de mon sujet de thèse. En effet, pas un colloque ne s'est déroulé sans que je reçoive le compliment déroutant : "vous avez un très beau sujet de thèse, vous savez ». Alors que j'entame actuellement un processus visant à faire publier ma thèse, ces remarques furent réitérés. Il me semble donc important d'insister sur le fait que le sujet de thèse sur 
lequel on choisit de travailler n'a rien d'anodin. Il doit être défini en fonction de nos centres d'intérêts, de nos compétences, mais aussi des dynamiques de la recherche et des évolutions d'un domaine. J'entends déjà siffler les accusations d'opportunisme scientifique et de libéralisation de la recherche. Le doctorat - et au-delà le métier d'enseignant-chercheur - est effectivement un exercice de tactique, non pas militaire, mais professionnelle. Il faut faire montre d'une faculté à la manœuvre, sans tomber dans les machinations et les manipulations. La nuance est importante. Peut-être faut-il le voir comme un jeu: déplacer ses pions au bon moment, trouver des alliés, prendre des risques en les mesurant, en n'oubliant pas le plaisir de l'émulation intellectuelle. Dans cette partie, la négociation est subtile entre logiques individuelles et intérêt collectif. La recherche de cet équilibre anime la vie du chercheur jusqu'au dernier moment. Tout au long de mon parcours, j'ai été touchée et inspirée par l'humanité et la générosité de quelques rares personnes, dans un écosystème, par ailleurs, sauvage, voire hostile, où le règne de la concurrence ne doit jamais être ignoré. J'ai eu beaucoup de chances de les trouver sur mon chemin. S'ils lisent ce texte, j'espère qu'ils se reconnaîtront.

\section{NOTES}

1. "Archives d'architectes en France. 1968-1998. Jeux d'acteurs et enjeux historiographiques autour de l'Institut français d'architecture ", thèse de doctorat en histoire de l'art de l'Université Bordeaux Montaigne et de l'École du Louvre soutenue le 29 novembre 2019 à la Maison de la Recherche de l'Université Bordeaux Montaigne et réalisée sous la direction de Gilles Ragot David Peyceré.

2. Yvan Jablonka, Histoire des grands-parents que je n'ai pas eus : une enquête, Éditions du Seuil, Paris, 2012.

3. Gilles Ragot, «L'historien, l'architecte et le politique. Du respect de la juste distance », Revue de l'art, 2014, $\mathrm{n}^{\circ} 186$ « Architecture du XX ${ }^{\mathrm{e}}$ siècle », p. 75-82.

4. Bruno Latour et Steve Woolgar, La vie de laboratoire: la production de faits scientifiques, Paris, France, la Découverte, 2008, 299 p.

5. Héloïse Lhérété, «La solitude du thésard de fond », Sciences humaines, n²30, octobre 2011, p. 48-51.

6. Voir les articles d'Olivier Bonfait sur le blog de l'Apahau : «Un nombre de postes ouverts dans l'enseignement supérieur en hausse", Blog de l'Apahau, 15 mars 2020 ; "Recrutement à l'université : un nombre de postes toujours insuffisant », Blog de l'Apahau, 15 avril 2016.

7. Richar Klein (dir), À quoi sert l'histoire de l'architecture ?, Hermann, Paris, 2018.

8. Isabelle Kabla-Langlois (dir.), «Vers l'égalité hommes-femmes? Chiffres clés. 2020 », Ministère de l'Enseignement supérieur, de la recherche et de l'innovation, p. 30: https:// www.enseignementsup-recherche.gouv.fr/sites/default/files/content_migration/document/ egalite_femmes_hommes_1151442.pdf.

9. Ibid., p. 36 


\section{AUTEUR}

\section{NINA MANSION}

Maître de conférences en Histoire et Cultures architecturales

École nationale supérieure d'architecture de Toulouse

Chercheure au Laboratoire de Recherche en Architecture LRA

Lauréate du Prix de thèse Valois du ministère de la Culture 